\title{
Analyse Économique Du Secteur Industriel Au Maroc
}

\section{El Ouahabi Sanae}

Doctorante en Sciences économiques, à la faculté des sciences juridiques, économiques et sociales à Tanger, Maroc

\section{Bousselhami Ahmed}

Professeur à la faculté des sciences juridiques, économiques et sociales,

Tanger, Maroc

Equipe de recherche en Economie, finance et développement

\section{Doi: 10.19044/esj.2018.v14n7p168 URL:http://dx.doi.org/10.19044/esj.2018.v14n7p168}

\begin{abstract}
In this study, we will focus on the different dimensions of performance, and make a descriptive analysis of the industrial sector's position in the national economy. We will also assess the clout and the performance of each industrial branch, through the measurement of the following indicators: the added value, the employment, the number of companies, the investment, the foreign trade, the comparative advantage and the degree of export commitment.

This research shows that the industry contributes the most to GDP with an average of about 19\% during the 1985-2016 period, followed by agriculture with $(15 \%)$, trade with $(12 \%)$, construction industry and public works with $(5 \%)$.

By industry, statistics show the predominance of the agri-food, textile, leather, mechanical, metal and electrical industries in overall industrial production over the period 1985-2016, while in terms of exports, the mechanical, metallic, electrical", and "textile and leather" branches are at the top of the list with a share of $27 \%$ for each.

Over the period of 1985-2014, the chemical and parachemical industry ranks first in terms of investments in the industrial sector with a $33 \%$ share, and thus promises significant growth prospects.

In terms of regions, figures show the predominance of the Grand Casablanca region, which accounts for $41 \%$ of companies created, $61 \%$ of national industrial production, $56 \%$ of industrial exports, and $50 \%$ of the total workforce employed during the 1985-2014 period. All this is happening with a remarkable rise of the Tangier-Tetouan region in recent years.
\end{abstract}

Keywords: Industry, branch, performance, production 


\section{Résumé}

Dans ce travail de recherche nous allons mener une réflexion sur les différentes dimensions de la performance et dresser une analyse descriptive de la place qu'occupe le secteur industriel dans l'économie nationale.

Nous allons également essayer d'évaluer le poids et la performance de chaque branche industrielle, à travers la mesure des indicateurs de la valeur ajoutée, de l'emploi, du nombre d'entreprises, de l'investissement, du commerce extérieur, de l'avantage comparatif et du degré d'engagement à l'exportation. L'étude révèle que l'industrie contribue le plus à la formation du PIB avec une moyenne d'environ 19\% durant la période 1985-2016, suivie de l'agriculture (15\%), du commerce (12\%), et du Bâtiment et travaux publics (5\%).

Par branche, les statistiques montrent la prédominance des branches "agroalimentaire", "textile et cuir" et "mécanique, métallique et électrique" dans la production industrielle globale durant la période 1985-2016, alors qu'en termes d'exportations, les branches "mécanique, métallique et électrique" et "textile et cuir" se placent en tête de liste avec une part de $27 \%$ chacune.

La branche chimique et parachimique est placée en première position en termes d'investissements engagés dans le secteur industriel durant la période 1985-2014 avec une part de 33\%, et augure ainsi des perspectives de croissance importantes.

Par région, les chiffrent révèlent la prédominance de la région du Grand Casablanca qui s'adjuge $41 \%$ d'entreprises créées, $61 \%$ de la production industrielle nationale, $56 \%$ des exportations industrielles et $50 \%$ du total des effectifs employés durant la période 1985-2014, avec une montée en puissance de la région de Tanger-Tétouan ces dernières années.

Mots clés : Industrie, branches, performance, production

\section{Introduction}

Omniprésente dans toutes les sphères de l'activité humaine, la performance joue un rôle primordial dans les analyses de la situation économique et financière des entreprises. Celles-ci doivent affronter les nouvelles caractéristiques de leurs environnements, telles que l'intensification de la concurrence, la mondialisation ainsi que l'internationalisation des marchés.

Dès lors, les entreprises doivent mesurer leurs progrès à partir d'une performance plus globale incluant, en dehors de la dimension économique, des dimensions sociales et environnementales.

Ainsi, divers critères sont retenus selon le point de vue adopté pour déterminer si un secteur est performant ou moins performant. Il s'agit en 
premier lieu du critère de l'efficience allocative, qui est celui que privilégie l'économiste, si on se réfère à la démarche de l'économie industrielle structuraliste.

Toutefois, les orientations des décideurs publics peuvent engager les agents économiques à adopter des critères de performance qui s'éloignent de l'efficience allocative au profit de grands objectifs de la politique économique, à savoir la capacité du secteur à créer des emplois, sa contribution à l'équilibre de la balance commerciale, à l'objectif de stabilité des prix et ses effets d'entraînement sur l'ensemble du système productif.

Dans ce travail, nous allons mener une réflexion sur les différentes dimensions de la performance, passer en revue les outils de mesure existants, présenter les variables explicatives de la performance, ensuite nous effectuerons une analyse économique de la place qu'occupe l'industrie par rapport aux autres secteurs de l'économie nationale.

Il s'agit également d'évaluer le poids et la performance de chaque branche industrielle, à travers les indicateurs de la valeur ajoutée, de l'emploi, du nombre d'entreprises, de l'investissement, du commerce extérieur et du degré d'engagement.

\section{Approches de la performance \\ Définition du concept de la performance}

La performance de l'entreprise est une notion subjective qui pourra avoir une multitude de définitions.

En effet, l'existence de plusieurs décideurs, chacun ayant des critères de jugement spécifiques à lui et distincts des autres explique l'absence de consensus sur la définition exacte du terme.

"Il y a autant de définitions de la performance que de parties prenantes à la vie de l'entreprise, ce qui signifie que la performance est une notion subjective. La performance est donc un concept tout à fait spécifique à un utilisateur de signaux descriptifs, soit de diagnostic du positionnement de cet état par rapport à une intention ou à un souhait d'informations" (Lebbas, 1996 ; Bourguignon, 1995).

Pour sa part, Elie Cohen (1994) propose une approche partielle de la performance de l'entreprise, qui permet de remettre en cause tous les aspects de l'activité, leurs caractéristiques propres et leurs contributions spécifiques aux coûts et aux résultats globaux de l'entreprise. Il propose un "diagnostic partiel de la performance", mais il se limite aux aspects financiers seulement.

La définition de la performance varie en fonction du domaine dans lequel elle est utilisée, ce qui implique par la suite une multitude de définitions. De ce fait, trouver une définition acceptable pour tout le monde est presque impossible, en raison de : 
- La multiplicité des domaines de recherche mettant 1'accent sur des processus et attributs différents.

- La subjectivité dans la détermination des critères d'évaluation de la performance.

La performance recouvre plusieurs concepts allant de l'efficacité, l'efficience à la productivité. Pour certains, elle représente l'ensemble des moyens, pour d'autres elle reflète les objectifs à réaliser. Cependant, les moyens et les objectifs sont aussi mal connus et peu maitrisés.

\section{Les mesures de la performance}

Il parait à priori préférable de se rattacher à une définition particulière de la performance avant de se pencher sur la question de sa mesure, qui dépend en grande partie de la dimension ou de l'approche considérée.

- Approche économique : cette approche repose principalement sur la notion d'objectif à atteindre. La performance économique est représentée par les indicateurs de croissance de la productivité, souvent utilisés de façon individuelle, de l'emploi, d'investissement et du chiffre d'affaires.

- Approche sociale: Issue des apports de l'école des relations humaines, cette approche met en évidence la dimension humaine de l'entreprise, en raison de son importance puisque l'atteinte des objectifs sociaux permet en quelque sorte d'aboutir aux résultats financiers et économiques souhaités par les entreprises.

En effet, l'entreprise doit satisfaire les attentes des travailleurs, en créant un climat favorable au travail et en assurant des salaires satisfaisants.

- Approche politique : Cette approche met en cause les deux approches citées ci-dessus, en se basant sur le constat que chacune des deux, fixe certains buts spécifiques à l'entreprise. Or, tout dirigeant peut concevoir la performance de manière différente des autres, ce qui implique un relativisme au niveau de la détermination des objectifs de l'entreprise.

C'est dire qu'il est impossible de déclarer de manière univoque que l'on se trouve face à un "bon" ou à un "mauvais" secteur.

Même si on précise la position de notre point de vue, des divergences peuvent exister entre des critères rattachés au même angle d'attaque, qui pourraient déboucher sur des affirmations différentes sur le même secteur, comme par exemple les critères du risque/ rentabilité, et la création d'emploi/ compétitivité internationale.

A cela s'ajoute la problématique liée à l'horizon temporel adopté, dans la mesure où un "bon" secteur pourra être considéré pour un investisseur un secteur capable de dégager des profits à court terme, alors que pour d'autres ça serait le secteur capable de dégager plus de bénéfices dans la durée.

C'est dire que l'introduction de la notion de la temporalité dans l'évaluation des performances requiert la nécessité de distinguer, dans 
n'importe quel indicateur de performance, une composante transitoire (associée, par exemple, à un choc conjoncturel) et d'une composante permanente qui est censée être déterminée par les caractéristiques structurelles du secteur.

Le secteur constitue le point d'ancrage des performances individuelles et l'environnement où s'affirment un certain nombre de facteurs structurels (les attentes des clients, les contraintes technologiques, les "forces de la concurrence"...) qui définissent un ensemble de menaces et d'opportunités partagé par l'ensemble des entreprises.

Certains de ces facteurs structurels agissent directement sur le potentiel de performance des entreprises : la croissance de la demande sur la croissance du chiffre d'affaires, l'intensité de la concurrence sur la rentabilité, le dynamisme innovateur en amont et la "proximité de la science" sur les gains de productivité et la vitesse de renouvellement des produits...

A cet effet, les caractéristiques structurelles du secteur déterminent en quelques sortes "l'espérance des performances" ou les perspectives d'évolution auxquelles peuvent s'attendre les entreprises qui appartiennent à ce secteur. Toutefois, la divergence des performances entre les entreprises du même secteur aurait être due dans une grande mesure à la capacité de chaque firme à exploiter le potentiel de l'environnement sectoriel et à ses avantages compétitifs.

\section{Structure et caractéristiques du secteur industriel marocain}

La part du secteur industriel dans le PIB n'a pas pu atteindre la place qui lui échoit dans l'économie marocaine et le niveau escompté par les différents plans de développement industriel lancés par le Maroc depuis l'indépendance.

Au cours des dix dernières années, le Maroc est à la recherche d'une nouvelle politique industrielle et s'est engagé dans des réformes ambitieuses visant à mieux s'intégrer dans l'économie mondiale, à faire de l'industrie le fer de lance de la croissance économique nationale dans les années à venir et à réduire la dépendance de l'économie des aléas climatiques.

\section{Part de l'industrie dans l'économie nationale Durant la période 1980 à 1990}

La part du secteur industriel dans le Produit intérieur brut (PIB) a été des plus importantes durant toute la période étudiée (1980-2016), avec une part atteignant même 22 pc en 1984 et 1985, en se situant en première position, suivi par celui de l'agriculture, du commerce et du Bâtiment et travaux publics.

La performance de l'industrie durant cette période s'explique par le plan de développement de 1981-1987, qui a stipulé un ensemble de mesures 
visant notamment à chercher une certaine relance de la croissance économique et à valoriser les phosphates avec le lancement du programme sidérurgique.

D'après les statistiques du Haut-commissariat au plan (HCP), on peut constater que l'industrie contribuait avec une part la plus importante dans le PIB par rapport aux autres secteurs avec une moyenne d'environ $21 \%$ sur la période 1980-1990, suivie du commerce (14,5\%), de l'agriculture (près de 14 pc) et du BTP $(4,5 \%)$.

Cette évolution de la valeur ajoutée industrielle est également attribuable à la stratégie de développement industriel envisagée pour la période 1988-1992 qui s'assignait pour objectif d'encourager les exportations, afin d'atténuer le lourd déficit de la balance commerciale et d'assurer une meilleure intégration du tissu industriel en vue d'atteindre un développement équilibré et de valoriser les ressources disponibles.

\section{Durant la période 1991 à 2001}

Durant cette période, il nous parait clairement que l'industrie continue à contribuer le plus à la formation du PIB par rapport aux autres secteurs avec une moyenne de plus de $18 \%$, même si cette part a diminué de 3\% par rapport à la période 1980-1990, suivie de l'agriculture (16,6\%), dont la part a augmenté de $2,6 \%$ par rapport à la décennie précédente et connu des fluctuations importantes pour atteindre son pic le plus haut en $1996(20,5 \%)$ et le plus bas en 2000 (13,3\%).

Quant à la part du commerce dans le PIB, elle s'est élevé à $12 \%$ durant la période 1991-2001, avec une baisse de 2,5\% par rapport à 1980-1990, alors que celle du BTP a atteint $4 \%$, soit presque le même niveau que la période précédente.

La phase de 1995-2005 a connu le début de relance de l'industrie nationale impulsée par la volonté des pouvoirs publics de renouer avec une croissance soutenue et un programme ambitieux de mise à niveau des entreprises, mais cette relance demeure en deçà des attentes, en témoigne sa contribution dans la formation du PIB qui reste depuis 1997 aux alentours de $17 \%$.

\section{Durant la période 2002 à 2016}

Durant cette période, l'industrie continue à se placer en tête des 4 secteurs mentionnés dans la figure 1 en termes de contribution au PIB avec une moyenne de plus de $17,7 \%$, soit presque le même niveau que la période 1991-2001, même si cette période a été marquée par des chocs extérieurs à cause de la crise économique internationale.

Pour sa part, la part de l'agriculture s'est établie à $13,4 \%$ en moyenne par an durant la période 2002-2016, soit une régression de 3,2\% par rapport à 
la décennie précédente, sous l'effet des conditions climatiques qui ont prévalu durant les campagnes agricoles de cette période.

Quant à la part du commerce dans le PIB, elle s'est élevée à 9,85\% en moyenne par an durant la période 2002-2016, soit le niveau le plus bas durant toute la période de l'étude.

Pour ce qui est de la part du secteur du BTP dans le PIB, elle a atteint 5,77\% par an durant 2002-2016, en hausse de 1,8\% par rapport à 1991-2001, un dynamisme qui s'explique par la réalisation des programmes de construction de logements et des grands chantiers d'infrastructure qui ont permis ainsi d'améliorer la contribution du secteur au PIB, en passant de 3,8\% en 1998 à 5,7\% en 2016.

Figure 1: Part de l'industrie dans l'économie nationale

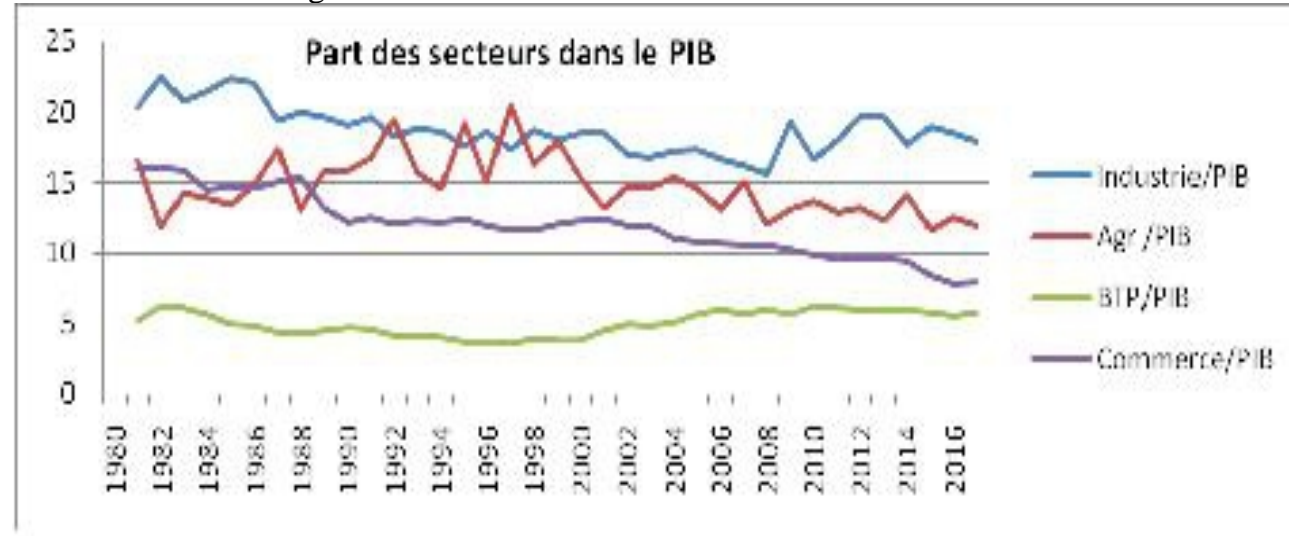

Source : HCP

Si on procède à une analyse du secteur industriel à part entière et le PIB par branche d'activité industrielle, on va constater une divergence de la contribution de chaque branche et ses potentiels de croissance.

Figure 2 : PIB par branches industrielles durant la période 1985-2016

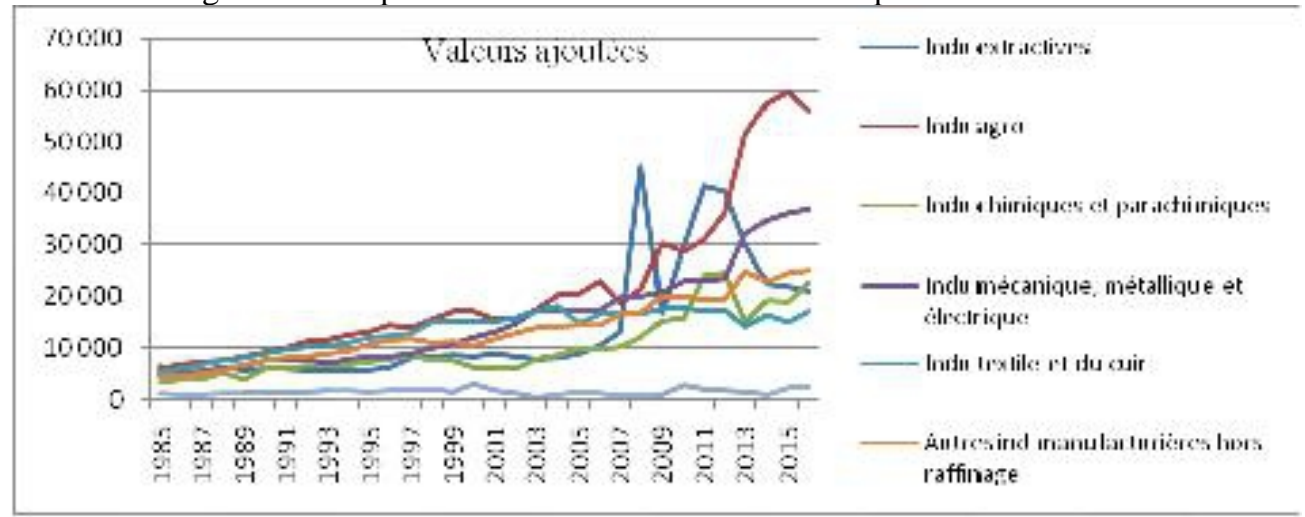

Source : HCP 
Ce graphique fait ressortir la contribution importante des industries agroalimentaires avec une tendance haussière depuis les années 1985 jusqu'à 2016.

Nous pouvons également remarquer les potentiels de croissance remarquables des industries chimiques et parachimiques, et celles mécaniques, métalliques et électriques sur lesquelles le Maroc mise dans son plan d'accélération industrielle pour renforcer sa valeur ajoutée industrielle, ses exportations et la compétitivité de son industrie.

Et de noter la croissance timide des industries du textile et du cuir même si le Royaume dispose des avantages comparatifs dans ce domaine, sauf qu'il manque d'une vision stratégique à même de renforcer son attractivité et sa compétitivité.

Quant aux autres industries manufacturières hors raffinage de pétrole, elles ont connu en général une progression, à l'exception des années 1998, 2000,2011 et 2014 où elles ont accusé des légères baisses, alors que le secteur du raffinage de pétrole et autres produits d'énergie a connu une hausse en dents de scie durant la période 1985-2016.

\section{La part de l'industrie dans la Formation brute de capital fixe (FBCF)}

Durant la période 1985-2016, la part moyenne du secteur industriel dans la FBCF globale a atteint $40 \%$, juste derrière le secteur du bâtiment et travaux publics $(51 \%)$, alors que les secteurs de services et de l'agriculture ont pris respectivement $7 \%$ et $2 \%$.

Pour sa part, le secteur de BTP occupe la première position en termes de FBCF avec des taux de croissance allant de 1\% à 19\%, en passant de 43571 millions de dirhams en 1998 à 144761 millions en 2016, soit un taux de croissance annuel moyen de $7 \%$.

La FBCF du secteur de l'agriculture a accusé des baisses considérables durant la période 1998-2016, à l'exception de quelques années où elle a enregistré une hausse, dont la plus importante est $26 \%$ en 2014 , alors que le secteur des services a connu une évolution en dents de scie durant cette période.

Figure 3 : La part des secteurs dans la FBCF totale

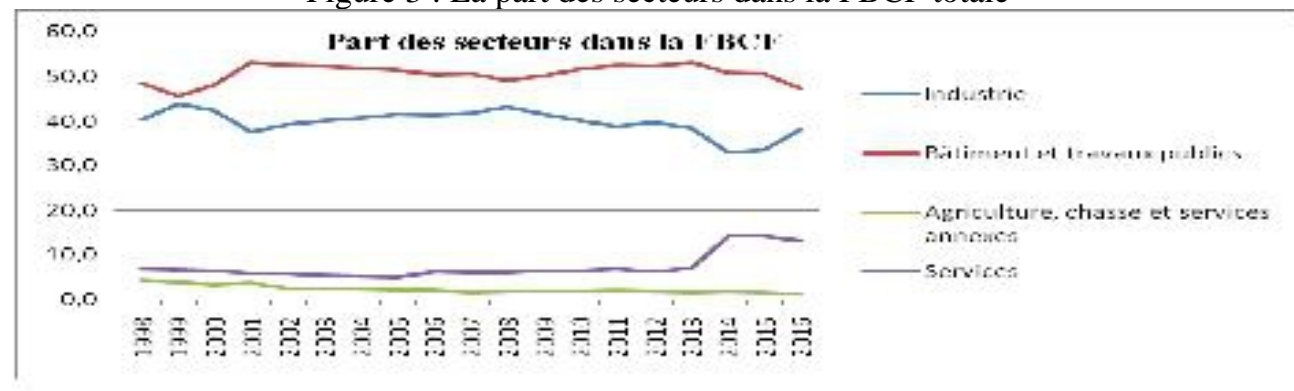

Source : HCP et calculs des auteurs 


\section{Taux d'investissement $=$ FBCF/Valeur ajoutée}

Le taux d'investissement du secteur de l'industrie (FBCF/VA) a connu une évolution fluctuante durant la période 1998-2016 avec un taux d'investissement annuel moyen de $63 \%$, en atteignant sa valeur la plus importante en 2007 (83\%), pour emprunter ensuite une pente baissière, qui est due notamment à la crise économique mondiale (figure 4).

Pour sa part, le secteur du BTP s'est hissé en première position avec un taux d'investissement annuel moyen de 80\% durant la période 1998-2016, alors que le taux d'investissement annuel moyen du secteur des services s'est établi à $11 \%$, avec une progression depuis 2013 pour atteindre son niveau le plus haut en $2016(23 \%)$.

Toutefois, le taux d'investissement annuel moyen du secteur de l'agriculture reste le plus faible avec seulement 3\% durant la période 19982016, avec une pente baissière, en passant d'environ 6\% en 1998 à seulement $2 \%$ en 2016 , ce qui reflète la faible part de l'investissement agricole dans la valeur ajoutée industrielle.

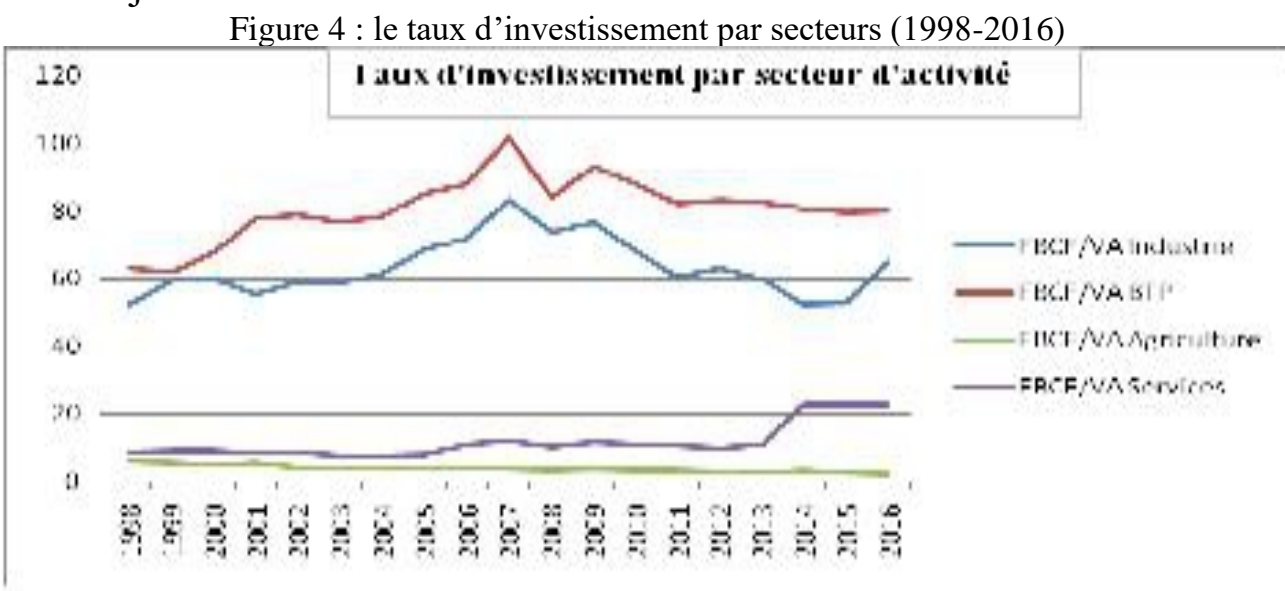

Source: HCP et calculs des auteurs

Grandeurs économiques caractérisant le secteur industriel Nombre d'entreprises industrielles

D'après les statistiques disponibles, le nombre d'entreprises des industries de transformation est en hausse continue depuis 1985, en atteignant son pic le plus haut de 8697 en 2004 avant d'entamer un trend baissier à partir de 2005 pour s'établir à 7687 entreprises en 2012. 
Figure 5 : Evolution du nombre d'entreprises industrielles durant 1985-2014

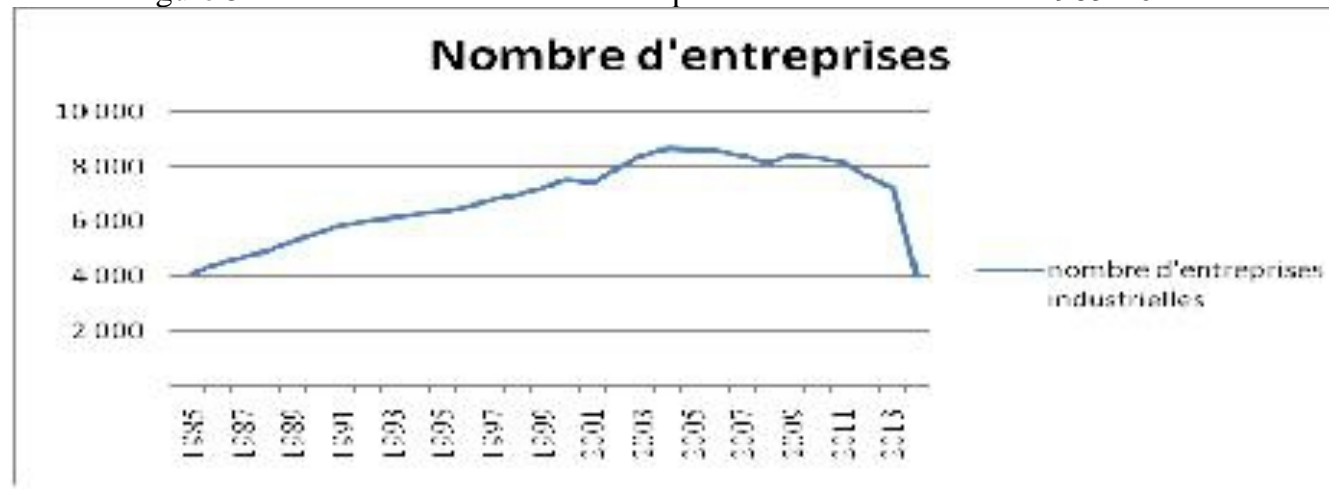

Source : Observatoire marocain de l'industrie

Ce graphique montre que le nombre d'entreprises industrielles a emprunté une pente haussière durant la période 1998-2004 avant d'entamer une trajectoire de baisse jusqu'au atteindre son pic le plus bas en 2014 avec une régression d'environ $45 \%$, ce qui témoigne soit du désengagement des opérateurs de quelques branches industrielles soit de la fusion de quelques entités pour avoir plus de poids sur le marché.

\section{Par branche industrielle}

Les industries chimiques et parachimiques se placent en tête de liste avec plus de $28 \%$ des entreprises des industries de transformation créées durant 1985-2014, suivies des industries agroalimentaires et du textile et cuir avec $25 \%$ pour chaque branche, des industries métalliques et mécaniques avec environ $19 \%$ et des industries électriques et électroniques avec près de $3 \%$ des entreprises.

Pour toutes les branches, il y a eu une baisse dans le nombre d'entreprises en 2008 en raison de la crise économique mondiale de 2008 qui sévit l'Europe, principal partenaire du Maroc.

On remarque aussi, à travers le graphique ci-dessus, que le nombre d'entreprises du secteur du textile et du cuir affiche une hausse timide depuis 1985 jusqu'à 2003 où il a atteint son maximum avec 2171 entreprises, avant de commencer à se dégrader pour arriver à seulement 839 entreprises en 2014, une situation qui reflète le désengagement des opérateurs de ce secteur, qui aurait être dû au manque de compétitivité face à une concurrence acharnée au niveau international.

Pour sa part, la branche agroalimentaire a accusé la baisse la plus importante du nombre d'entreprises en 2014 (-59\%), suivi des industries métalliques et mécaniques $(-48 \%)$, des industries électriques et électroniques $(-44 \%)$ et des industries chimiques et parachimiques $(-42 \%)$, ce qui nous 
pousse à s'interroger sur l'avenir desdites branches et des mesures nécessaires pour les redynamiser.

Figure 6 : Evolution du nombre d'entreprises par branches industrielles durant 1985-2014

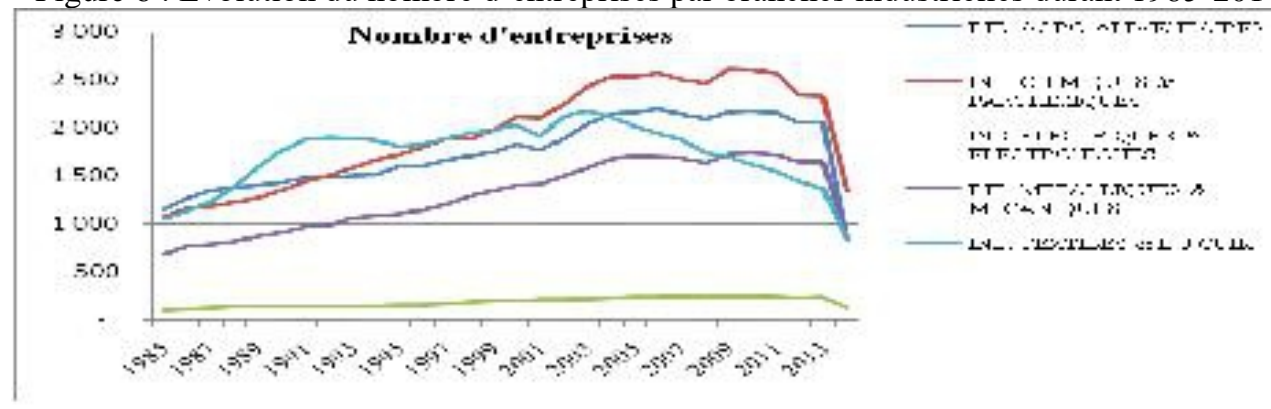

Source : Enquêtes annuelles du ministère de l'Industrie

\section{Evolution du nombre d'entreprises par région}

Par région, les données des enquêtes annuelles du ministère de l'industrie font ressortir une prédominance de la région du Grand Casablanca en s'adjugeant $41 \%$ du nombre total d'entreprises créées durant la période 1985-2014.

La région du Grand Casablanca est suivie par les régions de TangerTétouan (11\%), Fès-Boulmane (9\%), Rabat-Salé-Zemmour-Zaer et de Marrakech Tensift El Haouz et Chaouia Ouardigha (7\%).

Ces chiffres nous donnent une idée claire sur l'émergence de la région Tanger-Tétouan en deuxième position, ce qui constitue un signal fort de la volonté d'ériger cette région en pôle économique stratégique, étant donné qu'elle regorge des potentiels importants.

Figure 7 : Evolution du nombre d'entreprises par région durant 1985-2014

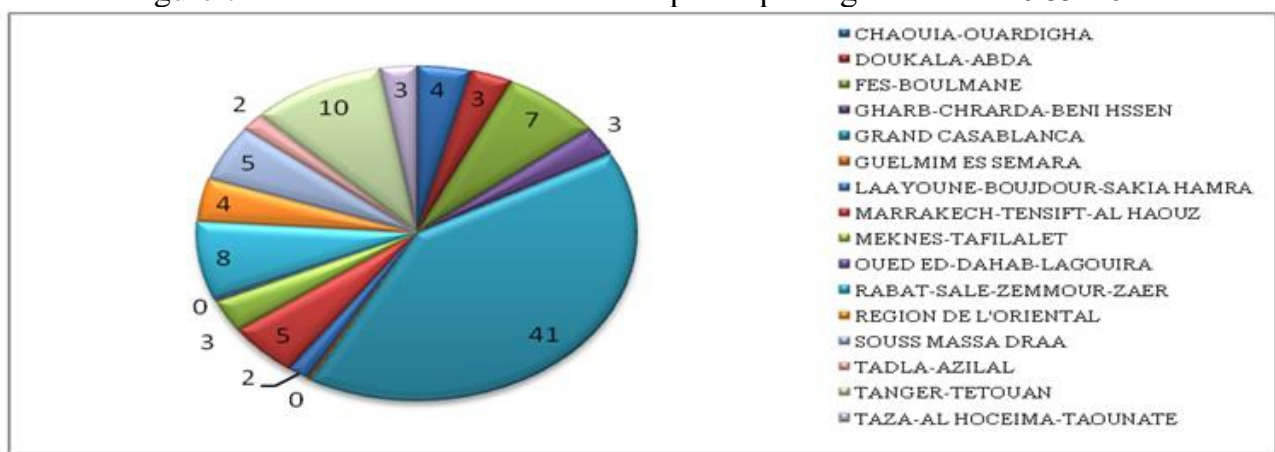

Source : Enquêtes annuelles du ministère de l'Industrie et calculs des auteurs

\section{Production industrielle}

Selon les chiffres du HCP disponibles, la production industrielle globale a connu durant la période 1985-2016 une progression continue, à l'exception de l'année 2009 où elle a accusé une baisse remarquable due à la 
crise économique de 2008, pour reprendre par la suite son rythme haussier, à l'exception d'une légère baisse en 2013 et 2015 (-3\%).

Figure 8 : Evolution de la production industrielle durant 1985-2016

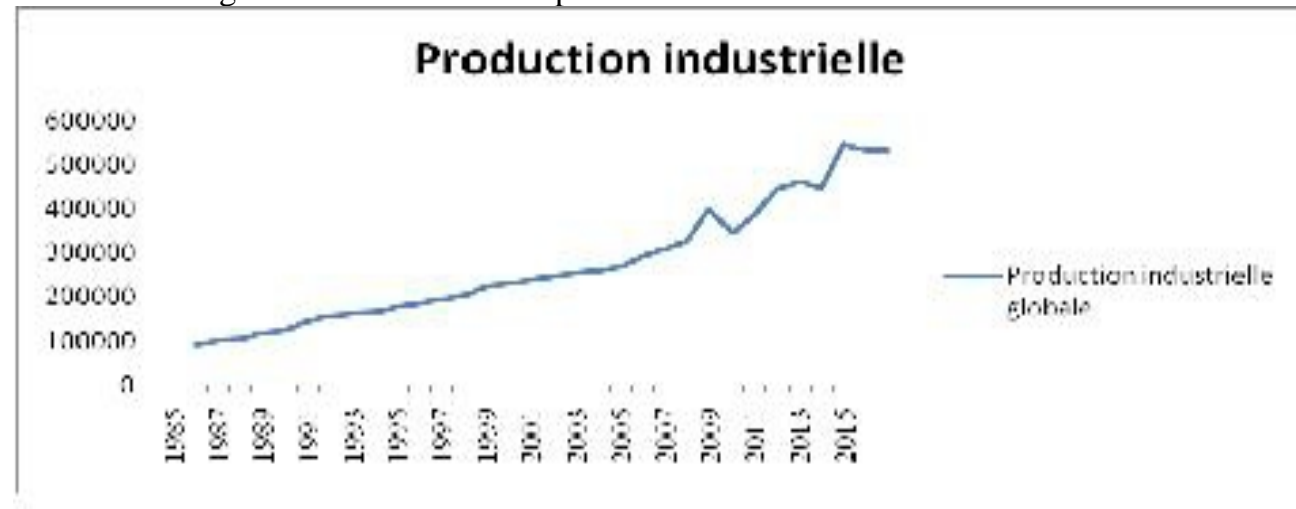

Source : HCP

\section{Par branche industrielle}

Les statistiques font ressortir une prédominance des secteurs agroalimentaire, du textile et cuir et du mécanique, métallique et électrique dans la production industrielle globale durant la période 1985-2016, avec des parts respectives de $31 \%, 15,7 \%$ et de $16 \%$, suivis de ceux des autres industries manufacturières $(14 \%)$ et de la chimique et de la parachimie (11\%).

Les industries extractives et le raffinage de pétrole ne représentent que $7 \%$ et $6 \%$ de la production industrielle globale durant cette période (figure 9).

Figure 9 : Evolution de la production industrielle par grands secteurs (1985-2016)

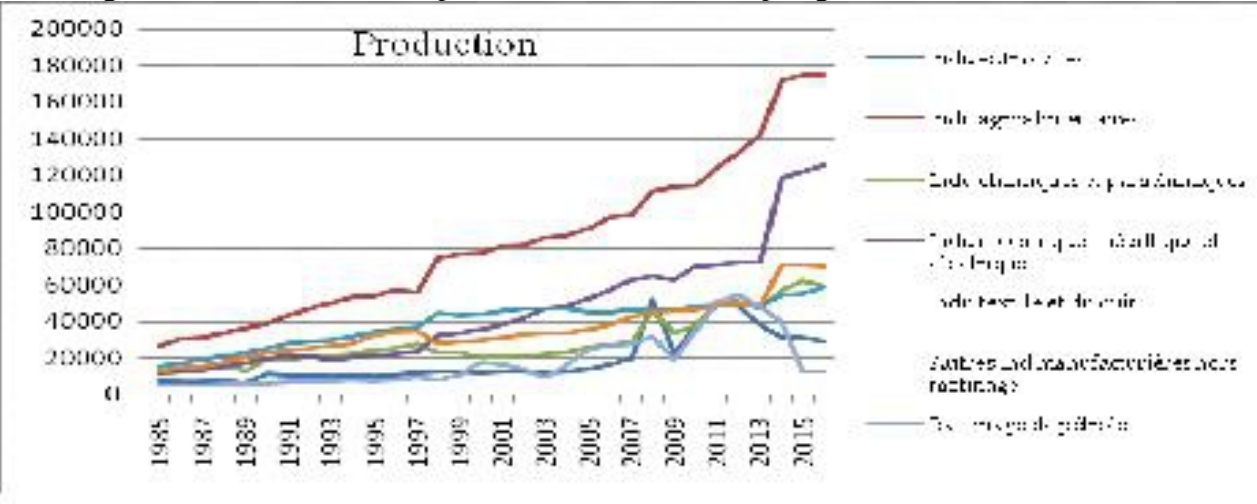

Source : HCP

\section{Evolution de la production industrielle par région}

Par région, les chiffres révèlent la prédominance de la région du Grand Casablanca en s'accaparant $61 \%$ de la production industrielle nationale durant la période 1985-2014. 
La région du Grand Casablanca est suivie par les régions de TangerTétouan (7\%), Rabat-Salé-Zemmour-Zaer et Chaouia Ouardigha (5\%) et de Doukkala-Abda et Souss Massa-Daraâ (4).

Figure 10 : Evolution de la production industrielle par région durant 1985-2014

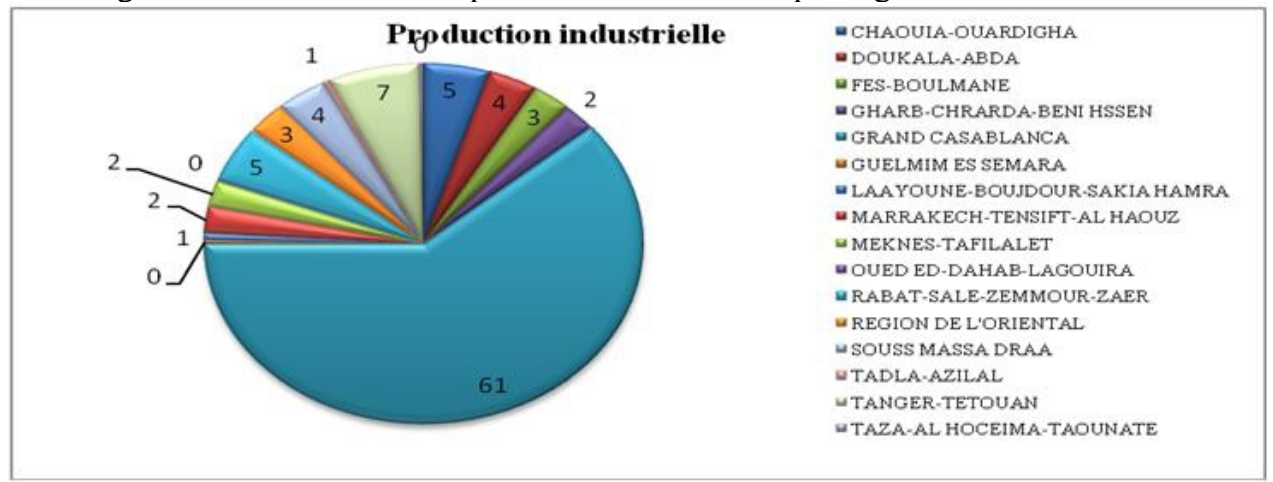

Source : Enquêtes annuelles du ministère de l'Industrie et calculs des auteurs

\section{Exportations industrielles}

Les exportations du secteur industriel durant la période 1998-2016 (selon la disponibilité des données) ont connu une tendance haussière, à l'exception d'une légère baisse de $1,2 \%$ en 2003 et 2013, alors que l'année 2009 a été marquée par une chute de $29 \%$ des exportations industrielles, qui aurait été due à la crise économique mondiale et ses répercussions sur les échanges commerciaux du Royaume (figure 11), pour renouer avec la croissance à partir de 2010.

A noter que la baisse des exportations du Royaume en 2009 s'explique en grande partie par le recul des dépenses touristiques non-résidents de $6,8 \%$ et le fléchissement des exportations des produits miniers $(45,1 \%)$, des produits agricoles $(13,6 \%)$ et du textile et habillement de 9,4\%.

Figure 11 : Evolution des exportations industrielles (1998-2016)

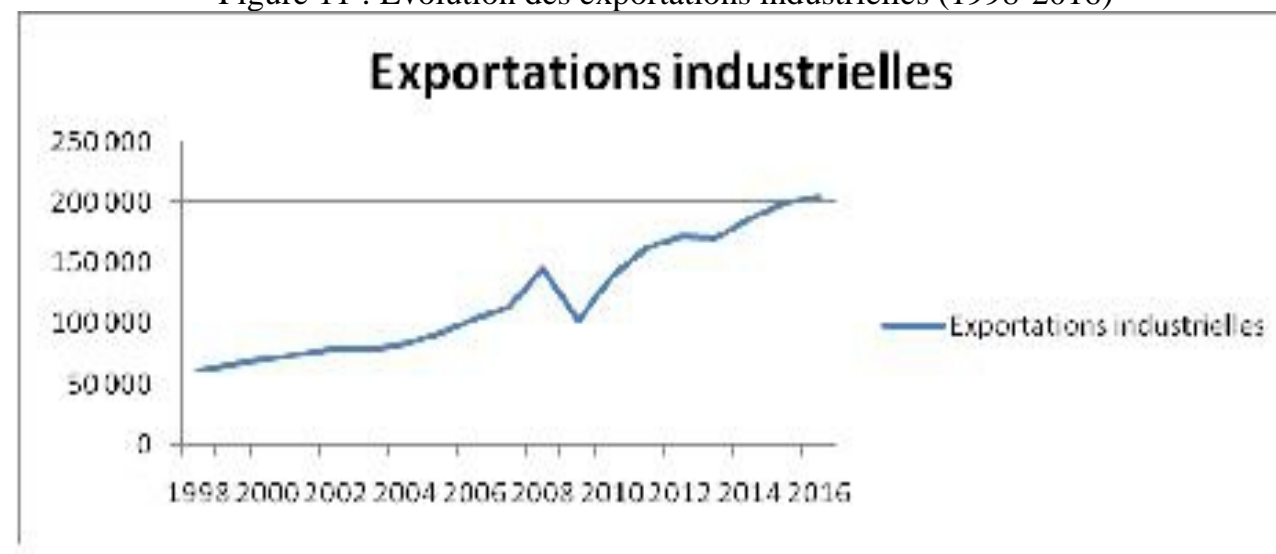

Source : HCP 


\section{Evolution des exportations par branche industrielle (1998-2016)}

Les chiffres font ressortir la prédominance des branches "mécanique, métallique et électrique" et du "textile et du cuir" dans les exportations industrielles globales avec une part de $27 \%$ chacune durant la période 1998 2016, suivies des industries chimiques et parachimiques (19\%), de l'agroalimentaire (13\%), des industries d'extraction $(8 \%)$, du raffinage du pétrole $(4 \%)$ et des autres industries manufacturières $(3 \%)$.

Les statistiques révèlent également une tendance haussière des exportations des industries mécaniques, métalliques et électriques, en particulier à partir de 2009, dans la mesure où elles ont enregistré une hausse de $43 \%$ en 2010, soit 40\% des exportations industrielles globales.

Ces chiffres laissent présager un avenir prometteur à la branche mécanique, métallique et électrique, sur laquelle le Maroc peut tabler pour développer davantage ses exportations.

Toutefois, l'agroalimentaire qui est le secteur le plus producteur avec $31 \%$ de la production industrielle nationale durant la période 1985-2016 ne contribue qu'avec $13 \%$ aux exportations industrielles nationales, ce qui peut être expliqué par le fait que cette production s'adresse en premier lieu au marché intérieur et témoigne de la faible compétitivité des produits agroalimentaires marocains et du manque de différenciation de la gamme des produits agricoles transformés.

Figure 12: Evolution des exportations industrielles par branche (1998-2016)

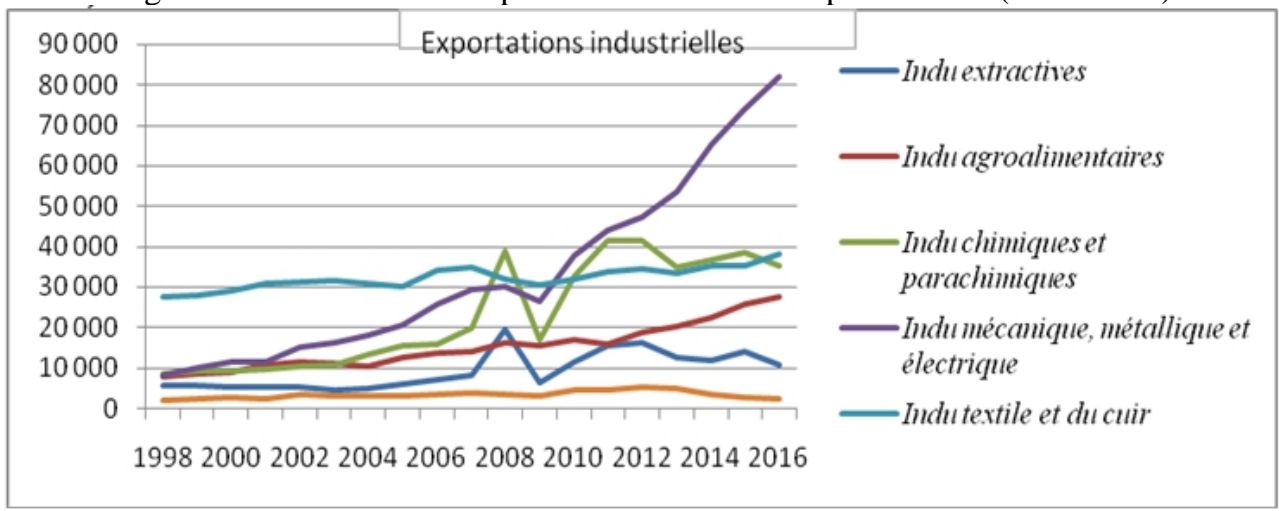

Source : HCP

\section{Evolution des exportations industrielles par région}

Les statistiques montrent que la région du Grand Casablanca s'accapare $56 \%$ des exportations industrielles nationales durant la période 1985-2014, suivie des régions de Tanger-Tétouan (14\%), Doukkala-Abda (7\%) et de Rabat-Salé-Zemmour-Zaer (5\%).

Les chiffres révèlent également une tendance à l'émergence de la région de Tanger-Tétouan, dont la part de ses est passée de 3\% en 1985 à plus 
de $30 \%$ en 2014, contre 44\% pour la région du Grand Casablanca en 2014, et ce en raison de l'installation de grandes entreprises multinationales.

Figure 13 : Evolution des exportations industrielles par région durant 1985-2014

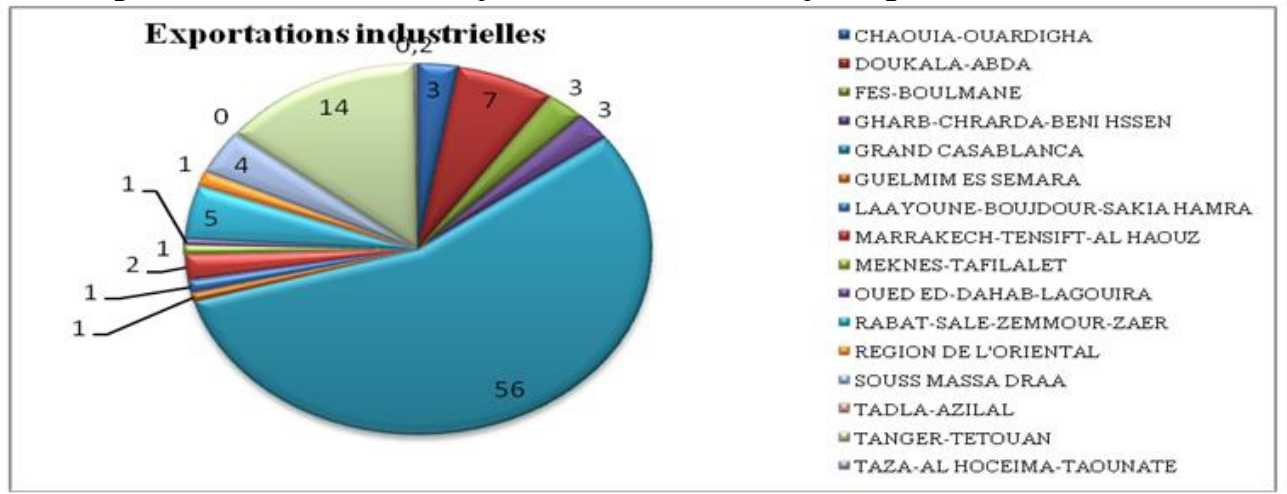

Source : Enquêtes annuelles du ministère de l'Industrie et calculs des auteurs

\section{L'investissement industriel}

L'investissement industriel a connu une évolution mitigée durant la période 1985-2014. Cependant, il a emprunté une trajectoire haussière à partir de 2011, pour atteindre son niveau le plus haut en 2014 avec une augmentation de $70 \%$, ce qui dénote de l'intérêt accordé de plus en plus à ce secteur stratégique pour l'émergence de l'économie marocaine.

\section{Par branche industrielle}

Les statistiques font ressortir une prédominance de la branche des industries chimiques et parachimiques en termes d'investissements engagés dans le secteur industriel durant la période 1985-2014 avec une part de 33\%.

Pour sa part, la branche des industries d'extraction minière vient en deuxième position avec une part de $23 \%$ des investissements industriels engagés durant la période 1985-2014, notamment en matière de recherche et d'exploration minière.

Le secteur de l'agroalimentaire s'est, quant à lui, accaparé $16 \%$ du total d'investissements industriels durant la période 1985-2014, suivi de celui des industries métalliques, mécaniques et électriques (13\%).

Cependant, la branche du textile et cuir s'est adjugée seulement $8 \%$ d'investissements industriels. 
Figure 14 : Evolution de l'investissement industriel par branches (1985-2014)

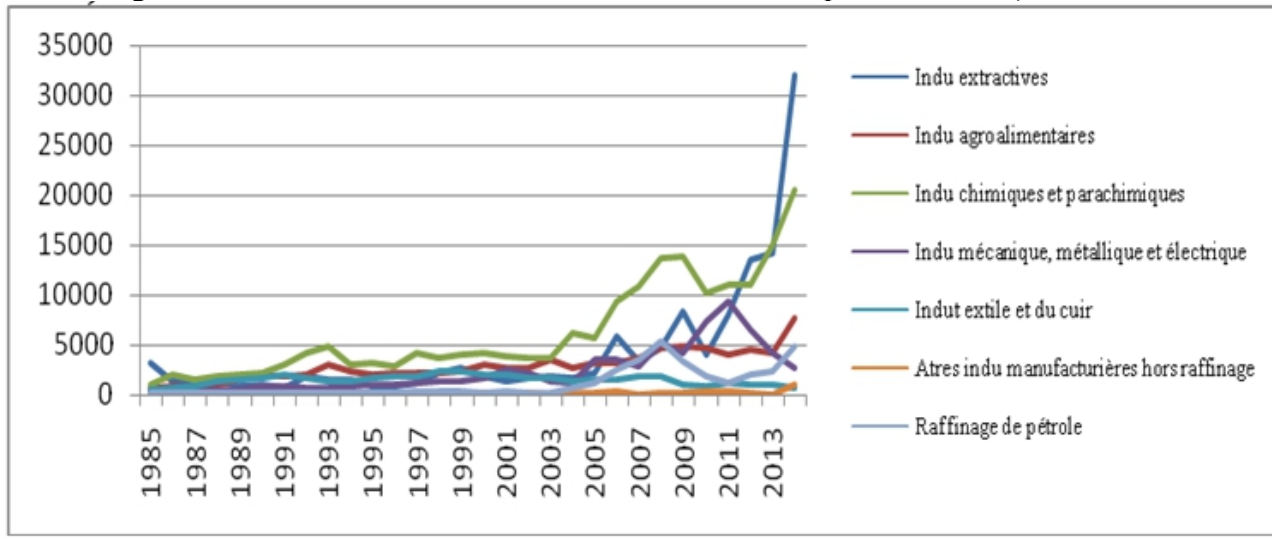

Source : Enquête annuelle sur les industries de transformation et ministère de l'Energie et des mines

\section{Evolution de l'investissement industriel par région (1985-2013)}

Par région, les chiffres font ressortir clairement la concentration des investissements industriels durant toute la période d'étude dans la région du Grand Casablanca avec une moyenne annuelle de $60 \%$ du total des investissements engagés dans les industries de transformation.

Les chiffres montrent également une montée en puissance de la région de Tanger-Tétouan, dans la mesure où sa part dans l'investissement industriel est passée de $5 \%$ en 1998 à $25 \%$ en 2011 , avant d'entamer une trajectoire baissière jusqu'au atteindre seulement 4\% en 2014.

Figure 15 : Evolution du cumul d'investissement industriel par région (1985-2014)

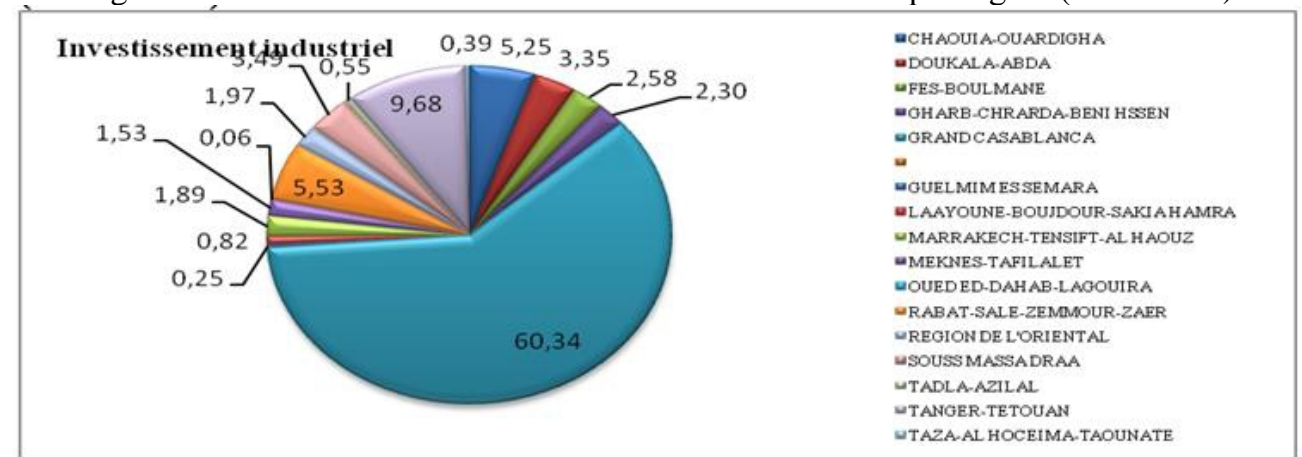

Source : Enquêtes annuelles du ministère de l'Industrie sur les industries de transformation et calculs des auteurs

\section{Emploi industriel}

L'effectif total employé par les industries de transformation a connu une hausse progressive durant la période 1985-1997 pour connaitre par la suite une évolution fluctuante jusqu'à s'établir à 597.441 employés en 2014. 


\section{Par branche industrielle}

Par branche, les chiffres montrent une prédominance du secteur du textile et cuir avec 39\% de l'effectif total employé dans les industries de transformation durant la période 1985-2014, mais sa part a connu une baisse progressive à partir de 2002 où elle a atteint sa valeur la plus importante, à savoir $46 \%$ des emplois industriels, en raison notamment de la baisse du nombre d'entreprises dans le secteur.

Le secteur du textile est suivi par l'agroalimentaire avec une part de $25 \%$ en 2014 , la chimie et la parachimie $(21 \%)$, les industries métalliques et mécaniques $(11 \%)$ et l'électrique et l'électronique (15\%). Le secteur de la chimie est classé en troisième position malgré qu'il est le deuxième en termes du nombre d'entreprises, qui peut s'expliquer par la nature du secteur qui est fortement capitalistique et se base dans une large mesure sur les nouvelles technologies et des machines sophistiquées.

Figure 16 : Evolution de l'effectif employé par branche industrielle durant 1985-2014

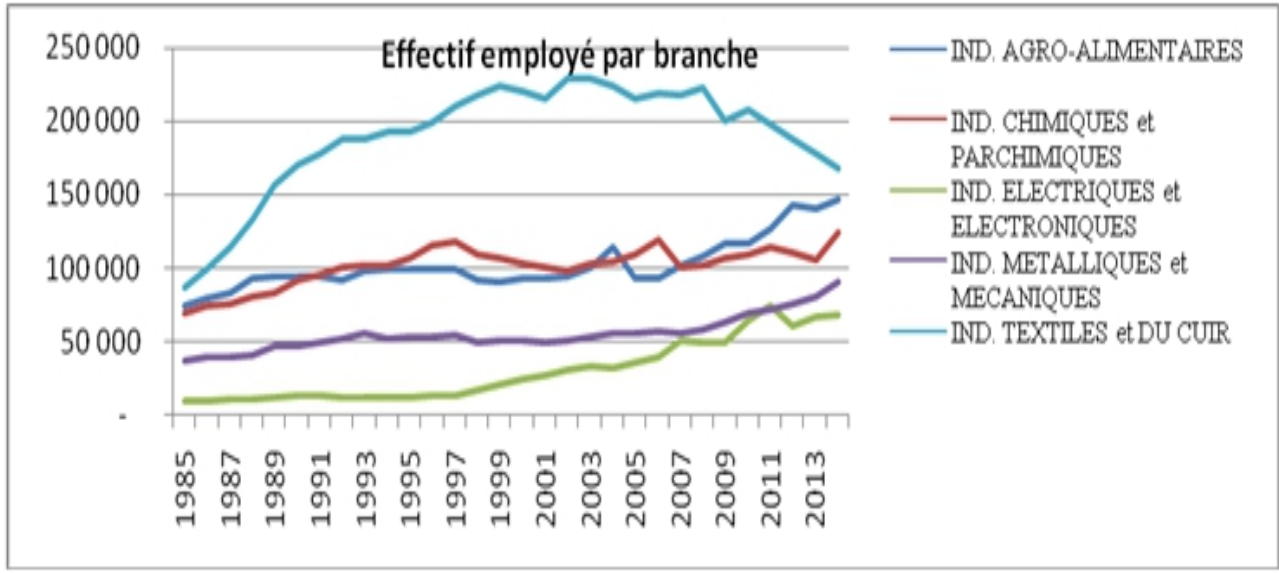

Source : Enquêtes annuelles sur les industries de transformation

\section{Evolution de l'effectif employé par région (1985-2014)}

Les chiffres révèlent la concentration de l'effectif employé durant la période 1985-2014 dans la région du Grand Casablanca avec une moyenne annuelle de $50 \%$ du total des effectifs employés dans les industries de transformation.

Cependant, cette part commence à se dégrader depuis 2005, en passant de $47 \%$ à $39 \%$ en 2014 .

Les chiffres montrent également une montée en puissance de la région de Tanger-Tétouan, dans la mesure où sa part dans l'effectif total employé est passée de $9 \%$ en 1998 à $20 \%$ en 2014. 
Figure 17 : Evolution de l'effectif employé dans les industries de transformation par région (1985-2014)

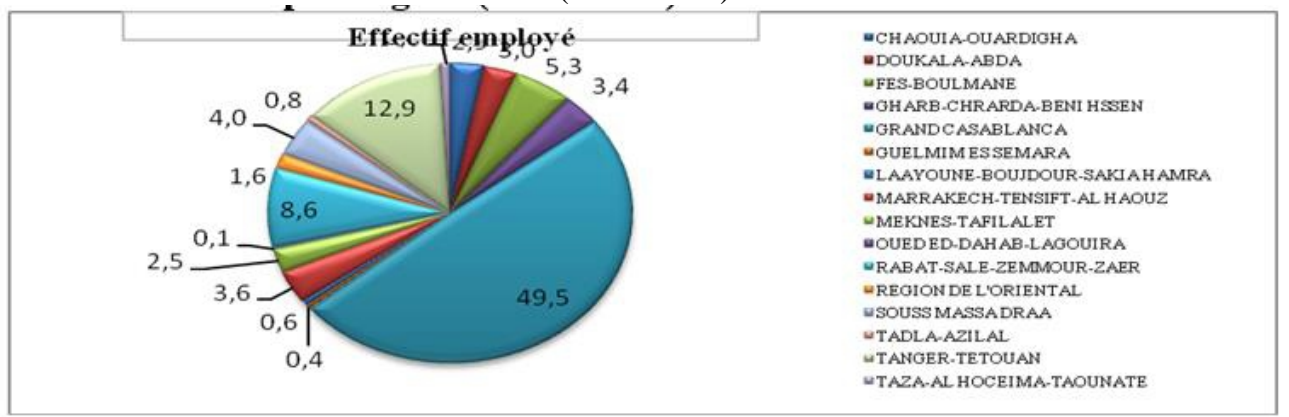

Source : Enquêtes annuelles du ministère de l'Industrie sur les industries de transformation et calculs des auteurs

\section{Le commerce extérieur}

\section{Le taux de couverture du secteur industriel (1998-2016)}

Taux de couverture $=$ Exportations / Importations

Selon les chiffres dont nous disposons sur les exportations et les importations du secteur industriel, le taux de couverture des importations industrielles par les exportations reste en dessous des $100 \%$, plus encore il a accusé une baisse en passant de 67\% en 1998 à 47\% en 2013, avant d'entamer une trajectoire haussière jusqu'au atteindre $57 \%$ en 2016.

Cette hausse peut s'expliquer par une montée en puissance de certaines branches industrielles, en affichant même un solde commercial positif ou proche de l'équilibre. C'est ce que nous allons analyser par la suite.

\section{Le taux de couverture par branche industrielle (1998-2016)}

Pour les industries extractives, son taux de couverture est en dessous de $100 \%$ durant toute la période $1998-2016$, plus encore ce taux a connu une dégradation en passant de 74\% en 1998 à $25 \%$ en 2013.

Cette situation s'explique par la forte dépendance du Maroc de l'étranger en matière des produits énergétiques et miniers, alors que ses exportations restent cantonnées dans des produits limités, notamment les phosphates et des produits miniers bruts.

Concernant le secteur du textile et cuir, ses exportations couvrent largement ses importations, en affichant même un excédent commercial. Cependant, son taux de couverture a connu une chute, en passant de $169 \%$ en 1998 à $116 \%$ en 2016, ce qui témoigne de la concurrence internationale acharnée.

Pour sa part, la branche de l'agroalimentaire a vu ses exportations couvrir largement ses importations jusqu'à 2006, où la tendance commence à baisser avec une hausse plus importante des importations par rapport aux exportations, avant d'emprunter une trajectoire haussière à partir de 2015. 
La branche de la chimie et la parachimie a, quant à elle, vu son taux de couverture passer de $78 \%$ en 1998 à $137 \%$ en 2008, puis il a baissé à $66 \%$ en 2009, à cause des répercussions de la crise économique mondiale sur les exportations marocaines. En 2010, les exportations de la branche ont renoué avec la croissance avec un taux de couverture de près de $110 \%$, de $114 \%$ en 2011 et de $115 \%$ en 2012.

S'agissant de la branche mécanique, métallique et électrique, son taux de couverture a augmenté progressivement durant la période 1998-2002, avant d'enregistrer une évolution mitigée pour emprunter une trajectoire haussière à partir de 2008 jusqu'au atteindre $50 \%$ en 2015 , ce qui témoigne de la montée en puissance de cette branche.

Figure 18 : Evolution du taux de couverture par branche industrielle durant 1998-2016

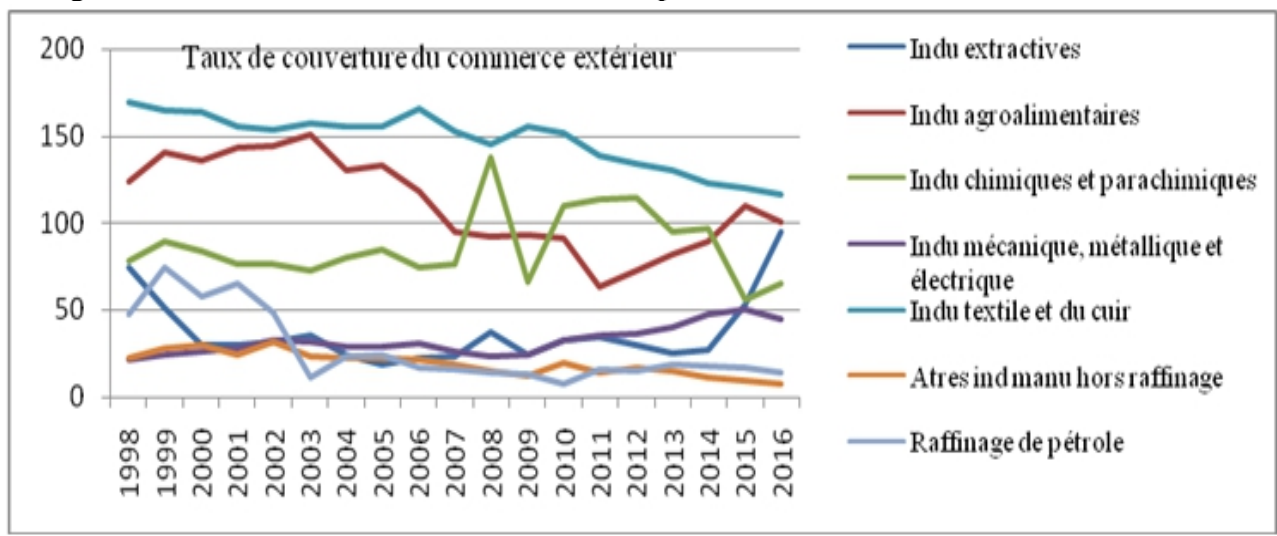

Source : HCP

\section{Le degré d'engagement : mesure de l'avantage comparatif}

Il existe une multitude d'indicateurs de spécialisation, mais nous allons utiliser l'indicateur du "degré d'engagement"2 qui rend compte de l'intensité de l'engagement du système productif national dans le secteur.

Un pays est dit engagé dans une activité dès lors que sa production excède le montant de sa consommation. Le degré d'engagement se mesure par le rapport de la production de la branche sur la consommation intérieure des produits de la branche:

\section{Degré d'engagement $=$ Production $/($ Production - Exportations + Importations)}

Un degré d'engagement significativement supérieur à 1 est considéré comme exprimant la spécialisation du pays dans l'activité étudiée.

2 «Evaluer les performances d'un secteur d'activité », Centre de recherche pour l'étude et l'observation des conditions de vie (Crédoc), Philippe Moati, cahier de recherche n 148 (septembre 2000) 
Inversement, un degré d'engagement significativement inférieur à 1 s'interprète comme une contre-spécialisation ou un désavantage comparatif.

Pour notre étude, la branche du textile et du cuir est la seule branche qui a affiché un degré d'engagement supérieur à 1 durant toute la période 1998-2016. Cependant, les chiffres font ressortir qu'à partir de 2006 le degré d'engagement de cette branche commence à se dégrader progressivement pour s'établir à 1,10 en 2016, ce qui nous pousse de s'interroger sur la compétitivité de cette branche et la structure de l'avantage comparatif sur lequel repose.

Vient en deuxième position la branche de l'agroalimentaire, qui a enregistré un degré d'engagement supérieur à 1 durant la période 1998-2006, pour commencer à partir de 2007 à se dégrader légèrement, mais tout en restant proche de 1.

Pour ce qui est du secteur de la chimie et de la parachimie, son degré d'engagement a connu une évolution mitigée durant la période 1998-2007, tout en se situant parfois à un degré proche de 1, pour dépasser la valeur 1 en 2008, 2010, 2011 et 2012, ce qui témoigne des perspectives prometteuses qu'affiche cette branche.

S'agissant de la branche de la mécanique, métallique et électrique, le Maroc affiche un degré d'engagement aux alentours de 0,50, atteignant même 0,62 en 2015, ce qui reflète la persistance de la dépendance du pays du marché extérieur.

Figure 19 : Evolution du degré d'engagement des branches industrielles durant 1998-2016

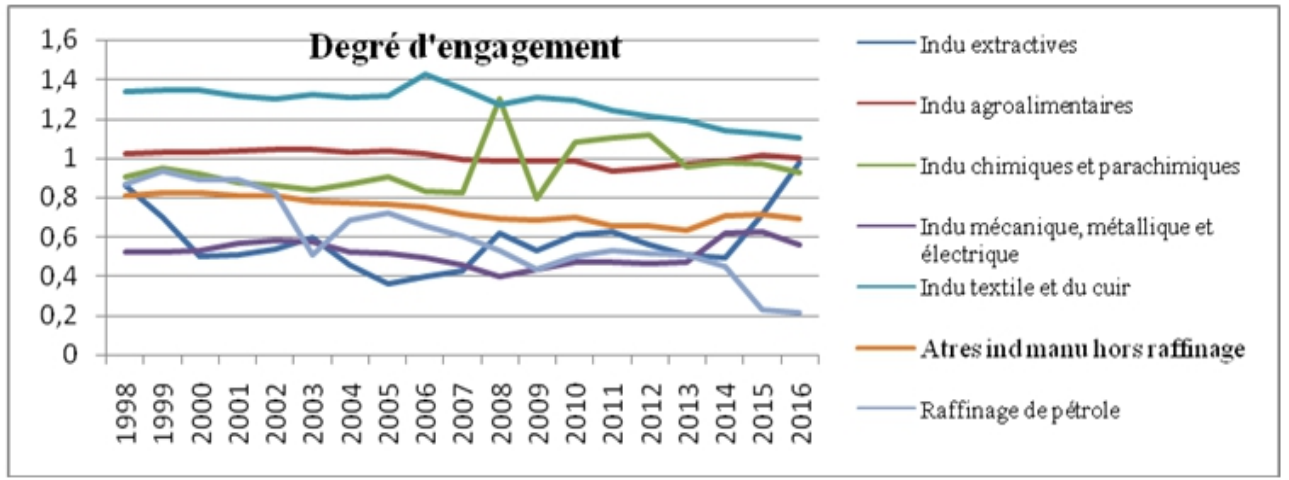

Source : HCP et calculs des auteurs

\section{Le taux d'exportation}

Les indicateurs étudiés ci-dessus peuvent être utilement complétés par le calcul du taux d'exportation.

\section{Taux d'exportation $=$ Exportations $/$ Chiffre d'affaires du secteur}

Le taux d'exportation mesure le degré d'engagement des entreprises dans la vente à l'étranger. Il peut conduire à un diagnostic très différent par rapport aux autres indicateurs précités. 
Pour notre cas d'étude, les chiffres font ressortir que les industries du textile et cuir se placent en tête de liste des branches des industries de transformation les plus engagées en termes d'exportation.

Le textile et cuir est suivi par les industries électriques et électroniques, dont le taux d'exportation a connu une évolution importante allant de $12 \%$ en 1985 à $76 \%$ en 2014 , ce qui témoigne de la performance croissante de cette branche.

Pour sa part, le taux d'exportation des industries chimiques et parachimiques est resté relativement stable en passant de $20 \%$ en 1985 à $25 \%$ en 2011 et $22 \%$ en 2014, ce qui signifie que le secteur a su maintenir sa position sur le marché international.

Concernant le taux d'exportation des industries agroalimentaires, il est resté modeste durant la période d'étude en passant de $9 \%$ en 1985 à $12 \%$ en 2014, malgré que son chiffre d'affaires dépasse largement celui des industries du textile et cuir, du fait que plus de $85 \%$ du chiffre d'affaires de la branche agroalimentaire est accaparé par le marché national.

S'agissant des industries métalliques et mécaniques, leur taux d'exportation a connu une évolution importante en passant de 5\% en 1985 à $37 \%$ en 2014, porté en particulier par les exportations de l'industrie automobile.

Figure 20 : Evolution du taux d'exportation des branches industrielles (1985-2014)

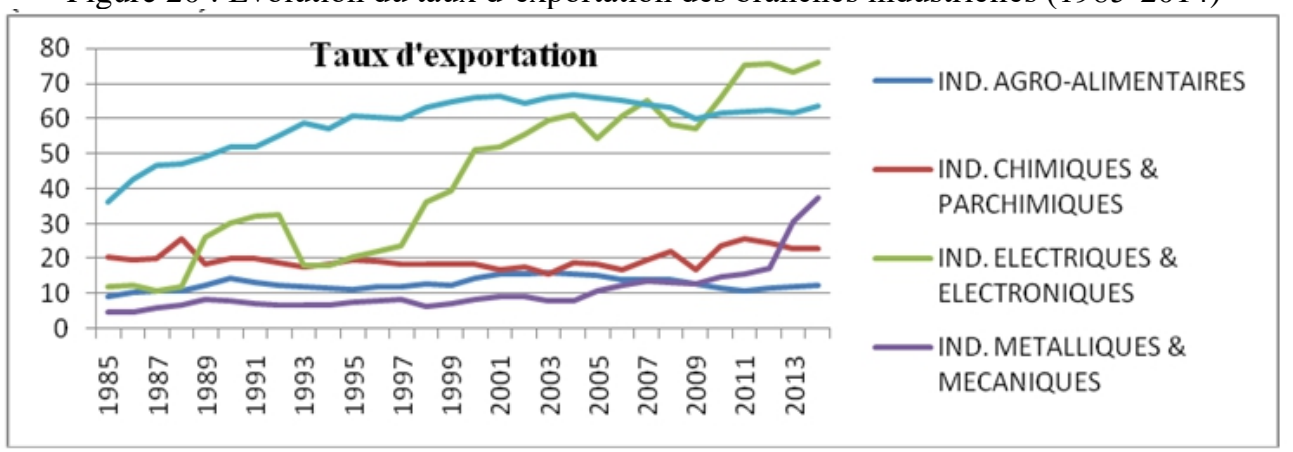

Source : Enquêtes annuelles du ministère de l'industrie et calculs des auteurs

\section{Conclusion}

L'analyse descriptive des indicateurs du secteur industriel révèle que l'industrie contribue le plus à la formation du PIB avec une moyenne d'environ $19 \%$ durant la période 1985-2016, suivie de l'agriculture (15\%), du commerce $(12 \%)$ et du Bâtiment et travaux publics (5\%).

La branche chimique et parachimique est placée en première position en termes d'investissements industriels engagés durant la période 1985-2014 avec une part de $33 \%$, alors qu'elle ne contribue qu'avec $11 \%$ à la production industrielle nationale. 
Malgré que les exportations de la branche du "textile et cuir" couvrent largement ses importations, son taux de couverture a connu une chute en passant de $169 \%$ en 1998 à $116 \%$ en 2016, ce qui témoigne de la nécessité de réguler cette industrie à même qu'elle soit capable d'affronter la concurrence en ayant une position de gagnant.

Pour sa part, la branche de l'agroalimentaire a vu ses exportations couvrir largement ses importations jusqu'à 2006, où la tendance commence à baisser avec une hausse plus importante des importations par rapport aux exportations, ce qui nous pousse à s'interroger sur la compétitivité de cette industrie et sa capacité à redynamiser ses exportations, tout en répondant à la demande intérieure.

S'agissant de la branche mécanique, métallique et électrique, son taux de couverture est passé de $21 \%$ en 1998 à 50\% en 2015, ce qui témoigne de la montée en puissance de cette branche durant ces dernières années et de ses perspectives prometteuses pour l'industrie marocaine.

A cet égard, le Maroc doit développer des avantages "compétitifs" forts plutôt que des avantages "comparatifs" dans ses branches industrielles, à travers la promotion de la recherche et développement dans le système productif industriel.

Par région, les chiffrent révèlent la prédominance de la région du Grand Casablanca en termes d'entreprises créées, de la production industrielle nationale, d'exportations industrielles et d'effectifs employés durant la période 1985-2014, avec une montée en puissance de la région de Tanger-Tétouan ces dernières années.

\section{References :}

1. Aglietta M. et Boyer R. (1982), Pôles de compétitivité, stratégie industrielle et politique macroéconomique, CEPII, N 8223.

2. Aglietta M. et Orlean A. et Oudiz G. (1980), L'industrie française face aux contraintes de change. Economie et Statistique, $\mathrm{n}^{\circ} 119$, Fév, pp. 35-63.

3. Carlton D. et Perloff J. (1998), Économie Industrielle, Edition DeBoeck.

4. CEPII, 1998, Compétitivité des nations, Economica, Paris.

5. Cohen E., Lorenzi J.-H. (2000), Politiques industrielles pour l'Europe, rapport du CAE, no 26, La Documentation française.

6. Combe E. (2002), La politique de la concurrence, Editions Repères La Decouverte.

7. Délégation à l'aménagement du teritoire et à l'action régionale (Datar). (2004), La France, puissance industrielle : une nouvelle politique industrielle par les territoires, La Documentation française. 
8. François J.-P. (1998), Innovation, croissance et emploi des entreprises industrielles, ronéotypé, Direction Générale des Stratégies Industrielles, SSI, 31 mars.

9. Gabszewicz, J.J. (2003), La concurrence imparfaite, Collection repères La Découverte, Paris, 2ème édition.

10. Humbert M. (1995), Elargissement des fondements théoriques standards des politiques industrielles, Revue d'économie industrielle Vol. 71.

11. Jaidi L. (1992), L'industrialisation de l'économie marocaine: acquis réels et modalités d'une remise en cause, édition du CNRS, Paris, 1992.

12. Moati P. 2000, Evaluer les performances d'un secteur d'activité, Crédoc, Cahier de recherche n 148.

13. Moati P. (1996), Méthode d'étude sectorielle, Cahier de recherche CREDOC, vol.2, ${ }^{\circ} 93$, septembre.

14. Moati P. (1997), Méthode d'étude sectorielle, Cahier de recherche CREDOC, vol.3, ${ }^{\circ} 109$, novembre.

15. Moati P., Chessa C., Pouquet L. (1998), Les déterminants sectoriels des défaillances d'entreprise dans l'industrie manufacturière, Cahier de recherche CREDOC, $n^{\circ} 121$, octobre.

16. Projet conjoint entre le Ministère de l'Industrie, du commerce, de l'énergie et des mines du Maroc et la Banque mondiale, Le secteur manufacturier marocain à l'aube du $21^{\text {ème }}$ siècle, résultats de l'enquête pour l'analyse de la compétitivité des entreprises.

17. Porter M.E. (1993), L'avantage concurrentiel des nations, Interéditions. Paris.

18. Tirole J. (1995), Théorie de l'organisation industrielle, Édition Economica. 